\title{
Patrón de alimentación e incorporación de alimentos complementarios en lactantes de una población de bajos recursos
}

\author{
Feeding pattern and incorporation of \\ complementary foods in infants from a \\ low- income population
}

\begin{abstract}
In Argentina the information about the characteristics of complementary feeding is scarce. The objective of the present study was to determine the age of incorporation and type of complementary foods in the diet of infants from a population of low economic resources. The study was carried out at the Forres District Hospital, Santiago del Estero, Argentina, using a structured questionnaire. Breastfeeding mothers were interrogated during the children's periodic control visits. A total of 240 surveys at 5, 6 and 7 months post-partum were made. The average age of incorporation of foods was of 4.4 months. The first introduced foods were purée (potato and pumpkin, with broth or soup), soup (with wheat grits) and apple. The percentage of children consuming some type of gruel at 5 months was of $51 \%$. At 7 months, 94\% consumed gruels and $62.5 \%$ had milk bottles. About $40 \%$ ate the family stew (vegetables, noodles and meat) and 30\%, a "mate" (Ilex paraguariensis) infusion with bread. An early incorporation of foods with inadequate energy and nutrients densities was observed. There was also an early ingestion of gluten and a high proportion of cow's milk consumption. This situation could affect the normal development of these children.

Key words: breastfeeding; complementary food; nutrients; energy density; cow milk.
\end{abstract}

\section{INTRODUCCIÓN}

El conocimiento de los patrones de introducción de alimentos constituye un punto fundamental para evaluar los factores asociados a inadecuaciones en la alimentación a fin de promover acciones oportunas de educación alimentaria. Los patrones inadecuados podrían traducirse tanto en situaciones de déficit como de exceso de nutrientes.

Son conocidos los beneficios inigualables que representa la alimentación al pecho tanto para la madre como para el niño, desde el punto de vista biológico, psicoafectivo, económico y social. Los niños amamantados por madres sanas y bien nutridas pueden alimentarse exclusivamente al pecho durante los primeros 6 meses $(1,2)$.

Sin embargo, muchos factores han llevado a la disminución de la lactancia materna. Entre ellos, la gran difusión de fórmulas lácteas que acompañaron el crecimiento de la industria, la gran disponibilidad de biberones y tetinas, la introducción temprana de otros alimentos, la administración
Sara M. Macias (1)

Silvia Rodríguez (1)

Patricia A. Ronayne de Ferrer (2)

(1) Instituto de Ciencia y Tecnologí deAlimentos. Facultad de Agronomía Agroindustrias. Universidad Nacional de Santiago del Estero, Argentina. (2) Cátedra de Bromatología. Facultad de Farmacia y Bioquímica. Universidad de Buenos Aires, Buenos Aires, Argentina.

Dirigir la correspondencia a: Dra. Sara M. Macias Instituto de Ciencia y Tecnología de Alimentos. Facultad de Agronomía y Agroindustrias. Universidad Nacional de Santiago del

Avenida Belgrano (S) 1912. Santiago del Estero. C.P. 4200, Argentina Tel.54 0385-4509528

E-mail: magui_macias@yahoo.com.ar

Este trabajo fue recibido el 16 de Octubre de 2012 y aceptado para ser publicado el 5 de Julio de 2013.

de líquidos azucarados, los que facilitaron la incorporación de la mujer al ambiente laboral (3).

La edad de introducción de alimentos complementarios es un momento de particular importancia en el desarrollo del niño. La dieta sufre un cambio radical, desde un único alimento (leche materna), con la grasa como principal fuente de energía a una variedad de alimentos que se requieren para cubrir las necesidades nutricionales. Esta transición se asocia no sólo con requerimientos de nutrientes crecientes y cambiantes, sino también con un rápido crecimiento, maduración fisiológica y desarrollo del lactante (4).

El momento óptimo para la introducción de la alimentación complementaria es objeto de controversias. Las recomendaciones de la OMS consideran los 6 meses como la edad adecuada para su inicio y definen a la alimentación complementaria como el período durante el cual se proveen otros alimentos o líquidos junto con la leche materna (5).

En nuestro país existe escasa información actualizada so- 
bre la incorporación de la alimentación complementaria, tanto en lo que hace al momento en que se realiza como al tipo de alimentos utilizados y a su composición, especialmente en la etapa de transición. Según la Encuesta Nacional de Nutrición y Salud, a nivel país se observó que más del 70\% de los niños incorporó algún alimento antes de los 6 meses de edad y que incluso el $25 \%$ lo hizo antes del cuarto mes de vida (6). Estudios realizados en la Argentina $(7,8)$ y en otros países latinoamericanos (9-16) también mostraron una introducción temprana de alimentos.

Los niños dependen de la alimentación que le proporcione su grupo familiar, la que estará condicionada por el contexto del mismo. Nuestro país tiene una historia que aúna las distintas latitudes, por lo cual existen diferencias interregionales y variaciones socio-económicas, culturales y en estilos de vida, así como en la ecología y el clima.

Todos estos aspectos tienen profunda repercusión sobre los hábitos de vida y alimentación de la población; la alimentación inapropiada resulta la cara visible de una compleja interrelación de los mismos. El 8\% de los niños de Argentina padecen de desnutrición crónica, producida por ingesta de alimentos inadecuados, por tipo y cantidad, así como hábitos de higiene deficientes. En la región del Noroeste, donde se encuentra la Provincia de Santiago del Estero, la proporción llega al 15,5\% (17).

La alimentación del niño, en especial en etapas tempranas de la vida es un aspecto fundamental para garantizar el adecuado proceso de crecimiento y desarrollo integral. Para evaluar la calidad de la dieta de un lactante, es importante considerar factores como son el tiempo de lactancia materna exclusiva y parcial, así como el tipo de alimentos sólidos y semisólidos incorporados.

\section{OBJETIVOS}

Determinar la edad de inicio de la alimentación complementaria y el tipo de alimentos incorporados en la dieta de lactantes, en una población de escasos recursos económicos.

\section{SUJETOS Y MÉTODOS}

Se utilizó un diseño de investigación cuantitativa, descriptiva de tipo prospectivo y transversal; considerando como unidad de observación las madres de niños de 5 a 7 meses y como unidad de análisis los niños. El tipo de muestreo fue no probabilístico, de tipo casual o incidencial. Se trabajó con mujeres que concurrieron al control pediátrico o vacunación de sus bebés en el Hospital Distrital de Forres, Departamento Robles, ubicado a $40 \mathrm{~km}$ de la capital de la Provincia de Santiago del Estero. Esta ciudad cuenta con una población estimada de 4.000 habitantes pero el radio de influencia municipal alcanza a varios parajes rurales, de donde llegan las personas que mayormente acuden a los servicios hospitalarios. La técnica empleada para la recopilación de los datos fue la encuesta y el procedimiento fue la entrevista. Se utilizó un cuestionario estructurado, y especialmente desarrollado. La entrevista fue llevada a cabo por personal entrenado perteneciente a la institución hospitalaria.

Se incluyeron únicamente mujeres de bajo nivel socioeconómico (NSE), en período de lactancia, aparentemente sanas, madres de niños nacidos a término. Se excluyeron las madres menores a 18 años y aquellos que presentaron partos prematuros (menos de 37 semanas de gestación) pues ésta sería una población con requerimientos nutricionales especiales desde su nacimiento. A fin de establecer el NSE e incluir sólo los hogares de familias correspondientes a NSE bajo, se utilizó un índice que considera el nivel de educación de la madre, el número de bienes domésticos y el hacinamiento del hogar (18).

Se tomaron 240 encuestas agrupadas en tres grupos de ochenta que correspondieron a los 5, 6 y 7 meses. El número de encuestas se consideró representativo de la población infantil atendida en el hospital. Estas encuestas se efectuaron con previo consentimiento escrito de las madres que aceptaron participar voluntariamente, luego de ser informadas sobre el objetivo del presente trabajo. Las preguntas fueron realizadas por el encuestador de forma clara, precisa y utilizando un lenguaje adecuado, en términos corrientes para la comprensión de las mismas, tendientes a que la mamá relate con sus propias palabras mientras el encuestador anota, abordando una sola idea por vez.

Se interrogó sobre los hábitos alimentarios del bebé, como la frecuencia de amamantamiento (número de repeticiones del acto de amamantar por día), y si esta alimentación era exclusiva o complementada por otros alimentos (leche de vaca y/o cabra, infusiones, papillas saladas, dulces o comida de la mesa familiar). Cuando esta respuesta era afirmativa se indagó sobre el momento de su incorporación y sobre el tipo y variedad de alimentos consumidos, sobre su consistencia y textura, combinación de alimentos, cantidad aproximada y frecuencia diaria de consumo de los mismos. Se trabajó sólo con testimonios en los que la entrevistada era la elaboradora de los alimentos para recopilar información detallada de la forma de preparación casera de los alimentos, mencionando los ingredientes usados. Los sesgos de información (omisiones, imprecisiones, errores introducidos por las encuestadoras, entre otros) se consideraron minimizados por el cuestionario estructurado, y sólo una persona adiestrada estuvo a cargo de su realización. Sólo unas pocas encuestas fueran desestimadas, y luego se incorporaron al grupo de estudio igual cantidad de nuevas encuestas, a fin de no modificar el número total.

Se consideró como alimentación complementaria la introducción de otros alimentos o líquidos (excluyendo la leche de vaca o fórmulas infantiles) junto con la leche materna. A fin de caracterizar el tipo de alimentación complementaria (oportuna, temprana o muy temprana) se utilizaron las definiciones de la Encuesta Nacional de Nutrición y Salud (6):

Alimentación Complementaria Oportuna (ACO): introducción de alimentos líquidos o sólidos cuyo fin es complementar la lactancia para cubrir los requerimientos nutricionales, y que no debe realizarse antes del sexto mes de edad del niño. Alimentación Complementaria Temprana (ACT): es la incorporación de alimentos líquidos o sólidos entre el cuarto y el sexto mes de edad del niño. Alimentación Complementaria Muy Temprana (ACMT): es la incorporación de alimentos líquidos o sólidos antes del cuarto mes de edad del niño. Se consideró Alimentación Complementaria Tardía (AC tardía) a aquella situación en la que los niños no habían incorporado otros alimentos, a excepción de la leche de vaca o fórmulas infantiles, a los 7 meses de edad.

El análisis estadístico de los datos se realizó mediante ANOVA.

\section{RESULTADOS}

Según las definiciones de la Encuesta Nacional de Nutrición y Salud (6) para caracterizar a la alimentación complementaria se observaron los siguientes porcentajes: ACMT: 15 \%; ACT: 50\%; ACO: 29\% y AC tardía: 6\%. El porcentaje de ACO fue algo menor pero similar al reportado por la Encuesta Nacional tanto a nivel país como en la región del NOA (31 
y $34 \%$ respectivamente); por otra parte, las proporciones de ACT y AC tardía fueron más elevadas, mientras que la ACMT mostró valores más bajos.

La frecuencia de comidas osciló entre 1 y 2 por día. A los 5 meses 58\% de los niños consumían una comida (almuerzo), porcentaje que bajaba a $49 \%$ en el $7^{\circ}$ mes, cuando el $51 \%$ consumían almuerzo y cena. Habitualmente las papillas eran de textura blanda, preparadas como puré.

En la figura 1 se muestra el tipo de alimentos que consumían los niños, separados en grupos de 5, 6 y 7 meses de edad. Se observa que a los 5 meses el 51,25\% de los bebés ya ingerían otros alimentos, además de leche, y que al sexto mes lo hacía el $82,5 \%$ de los niños. Es notorio que al quinto mes sólo el $22,5 \%$ de los niños encuestados se alimentaban en forma exclusiva con leche materna y al sexto mes $8,75 \%$. Se observó que a los 6 meses de vida, 17,5\% de los bebés no habían incluido aún alimentos sólidos o semisólidos, cifra que se redujo a $6,25 \%$ a los 7 meses. Con relación al consumo de leche no materna (leche de vaca o fórmula), las encuestas indicaron que aproximadamente $20 \%$ de los niños comenzó a ingerirla desde su nacimiento.
En la tabla 1 se observa que mientras la frecuencia de amamantamiento (número de veces que el bebé era amamantado en el día) tendía a disminuir de los 5 a los 7 meses de edad, el número de biberones de leche suministrados y la cantidad de leche no variaban mucho y era aproximadamente de $380 \mathrm{ml}$ diarios que se sumaban a la leche materna. Para ninguna de las variables se encontró diferencia significativa entre los grupos, posiblemente porque los niños ya consumían semisólidos desde antes de los 5 meses.

En la figura 2 se detalla la proporción de niños que incluyeron alimentos diferentes a la leche entre el quinto y séptimo mes. En la misma se observa la elevada proporción de niños que a temprana edad ya habían incorporado otros alimentos sólidos y semisólidos en su dieta.

Al analizar las encuestas y evaluar estadísticamente los resultados se determinó que la edad promedio de incorporación de papillas fue 4,4 \pm 1,1 meses (mediana $=4$ ). También se determinó que los primeros alimentos incorporados (denominados papillas) fueron puré (papa y zapallo), sopa (con sémola) y manzana (también banana y/o naranja, en menor proporción). Los niños que consumían una comida diaria in-

\section{FIGURA 1}

Tipos de alimentos (\%) que consumían los bebés de 5, 6 y 7 meses.

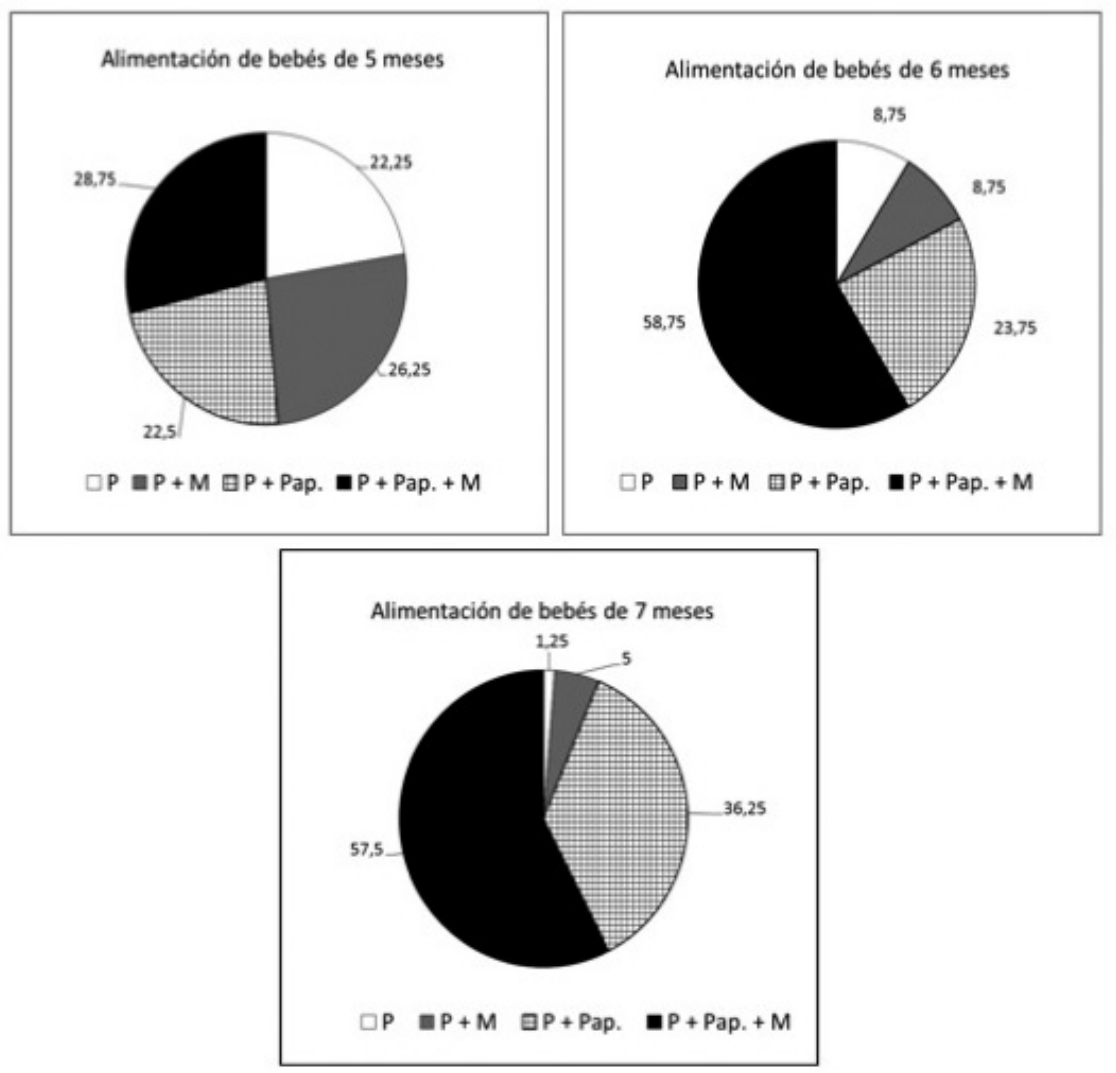

P: pecho; $P+M$ : pecho y mamadera; $P+P a p:$ pecho más papilla; $P+P a p+M$ : pecho, papilla y mamadera. 
gerían alrededor de 65 a 75 gr de alimento, mientras que los que consumían dos, ingerían alrededor de $150 \mathrm{~g}$.

En cuanto al consumo de papillas saladas y dulces, se observó que las madres optaron por brindar a sus hijos preferentemente papillas saladas con respecto a las dulces, proporción que se mantuvo en el tiempo. En la figura 3 se especifican los alimentos consumidos por los niños que incorporaron alimentación complementaria. Un elevado porcentaje de niños consumía papillas ricas en almidones con escaso aporte de nutrientes esenciales. También se observó que en muchos casos se reemplazaba la leche con infusiones tales como el mate cocido. La incorporación de carnes (pollo o vaca) en las papillas fue progresiva, para aproximadamente la mitad de los encuestados. Asimismo, es de destacar que muchos niños se incorporaban a la mesa familiar desde los 5 meses de edad, siendo el guiso uno de los alimentos más frecuentemente consumidos. Habitualmente el guiso se pre- paraba con arroz o fideos en una salsa (tomate, cebolla, aceite y condimentos) y algo de carne (cortes económicos de vaca o pollo). Frecuentemente a los bebés no les daban la carne, sino el resto de ingredientes aplastado con el tenedor.

\section{DISCUSIÓN}

Leche materna y no materna

La lactancia materna exclusiva durante los primeros seis meses de vida reduce la mortalidad infantil vinculada a las enfermedades comunes de la infancia y a la desnutrición (19). El apoyo durante el embarazo en la planificación de la lactancia materna es vital para el éxito.

La Encuesta Nacional de Nutrición y Salud (6) indicó un alto porcentaje de lactancia materna exclusiva en el primer mes de vida (el 95,4 \% de los niños inician su alimentación con pecho materno. Luego hay un descenso paulatino de la proporción de niños amamantados: al año alrededor de 50\%

\section{TABLA 1}

Tendencia central y variabilidad referida a la frecuencia de amamantamiento, número de biberones y cantidad de leche diaria consumida por bebés de 5, 6 y 7 meses.

\begin{tabular}{cccc}
\hline Meses de vida & $\begin{array}{c}\text { Frecuencia de } \\
\text { amamantamiento } \\
\mathrm{x} \pm \mathrm{DE}\end{array}$ & $\begin{array}{c}\text { No biberones diarios } \\
\mathrm{x} \pm \mathrm{DE}\end{array}$ & $\begin{array}{c}\text { Ingesta diaria total de } \\
\text { leche no materna (ml) } \\
\mathrm{x} \pm \mathrm{DE}\end{array}$ \\
5 & $6,5 \pm 2.6$ & $2,3 \pm 1.1$ & $382 \pm 142$ \\
6 & $6,2 \pm 2.4$ & $2,4 \pm 0.9$ & $381 \pm 129$ \\
7 & $5,6 \pm 2.3$ & $2,4 \pm 1.4$ & $370 \pm 153$ \\
\hline Diferencias significativas entre los grupos por ANOVA
\end{tabular}

Diferencias significativas entre los grupos por ANOVA

Porcentaje de niños que incluyeron alimentos diferentes a la leche (papillas), a los diferentes meses de vida.

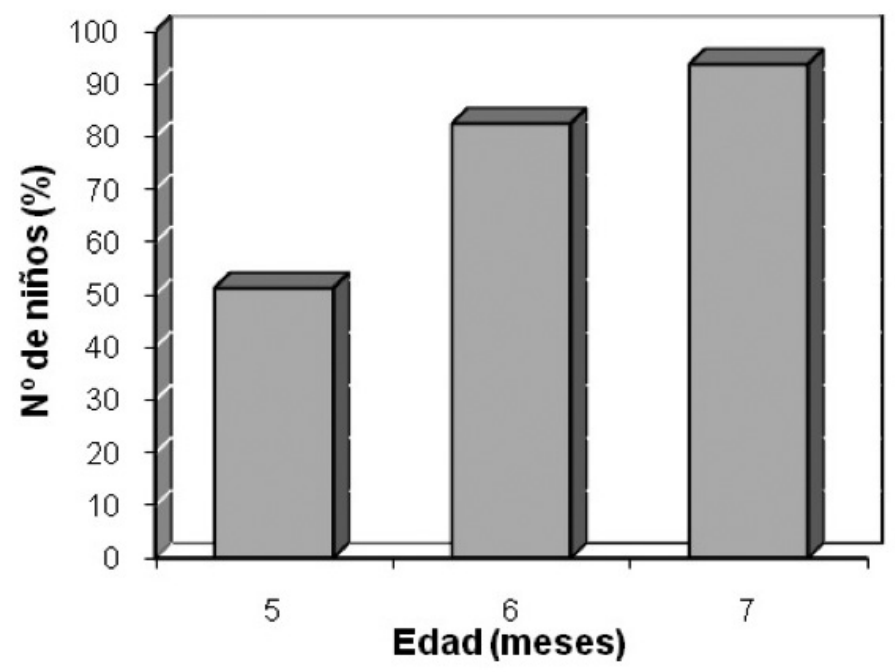


continúa siendo amamantado y a los dos años sólo 25\%. Estudios realizados en la ciudad de Córdoba (7) revelaron un alto porcentaje de lactancia materna exclusiva en el primer mes de vida con una caída brusca al tercer mes. En este estudio la frecuencia de amamantamiento diario fue un poco mayor a la reportada en otros trabajos (20).

Se observó que un porcentaje importante de los bebes amamantados $20 \%$ introdujeron en su dieta leche de vaca o fórmula desde su nacimiento, lo que hace suponer que se desplazó en parte la lactancia materna, tan importante en este período. El aporte necesario de factores de defensa y nutrientes podrían no ser suficientes, para cubrir las necesidades del niño, ya que la leche materna es la única que brinda todos los elementos necesarios para el normal desarrollo en esa etapa (19). En los lactantes se ha asociado la introducción temprana de leche de vaca, como uno de los factores que explicaría la alta incidencia de anemia en este grupo $(21,22)$. Asimismo, una dieta rica en proteínas y electrolitos provenientes de leche de vaca, en los primeros meses de vida conduce a una carga renal de solutos excesiva que puede afectar al riñón y la salud cardiovascular a largo plazo (23).

Nuestros resultados coinciden con los de la Encuesta Nacional de Nutrición y Salud (6), donde se menciona altos porcentajes de niños menores de 6 meses que incorporan leche de vaca tempranamente. Sin embargo, las medianas de ingesta de leche no materna para el grupo de niños amamantados fueron superiores a las referidas en dicha Encuesta $(115 \mathrm{ml}$ para niños de 6 a 8 meses).

\section{Papillas consumidas}

En este estudio la edad promedio de incorporación de papillas fue 4,4 meses. Un alto porcentaje de encuestados el inicio de la alimentación complementaria (puré de verduras y frutas) ocurrió a partir de los 3 meses de edad. Estos resultados concuerdan con datos obtenidos tanto a nivel provincial
(7) como nacional (6). A nivel nacional se observó que en los hogares más pobres la proporción de niños que recibieron alimentos antes de los 4 meses fue más elevada que en los hogares más favorecidos económicamente (6). Esta situación podría estar relacionada con prácticas de crianza ligadas a hábitos alimentarios familiares y/o modelos culturales locales. Por el contrario, en la Encuesta Nacional de Nutrición realizada en México (10) se observó que aunque la introducción precoz de alimentos era habitual (el 75\% de los niños incorporaron frutas y vegetales antes de los 6 meses), era más frecuente en familias de mejores condiciones habitacionales, residentes en áreas urbanas, y en hogares no indígenas. La introducción tardía de alimentos complementarios era más común en áreas rurales y entre familias más pobres y fue particularmente tardía en hogares indígenas.

Según la Encuesta Nacional de Nutrición y Salud (6), 68\% de los niños incorporó algún alimento antes de los 6 meses de edad. Estas cifras están sobre las registradas en Uruguay (20) donde 53,3\% había incorporado alguna comida antes de esa edad y muy alejadas de las de Chile, donde el porcentaje de lactantes amamantados que habían incorporado alimentos sólidos estaba alrededor del 7\% (24). Otros estudios en Argentina señalaron porcentajes de 24\% (8); en este estudio fue $65 \%$.

Publicaciones de Cuba, Brasil, Venezuela, Colombia, México y Bolivia $(9,11,12,14-16)$ mostraron pautas de incorporación de alimentos temprana o muy temprana.

En este estudio los primeros alimentos incorporados fueron puré (papa y zapallo), sopa (con sémola) y dentro del grupo de frutas, manzana principalmente. En todos los meses predominó el consumo de papillas saladas sobre las dulces, siendo una posible causa el acceso limitado de este sector poblacional a las frutas frescas. Estos resultados se asemejan a los observados en la Encuesta de Lactancia, Estado Nutricional y Alimentación Complementaria realizada en Uruguay (20) donde la primera comida que se le dio a probar al niño

Principales alimentos consumidos por bebés de 5, 6 y 7 meses de edad.

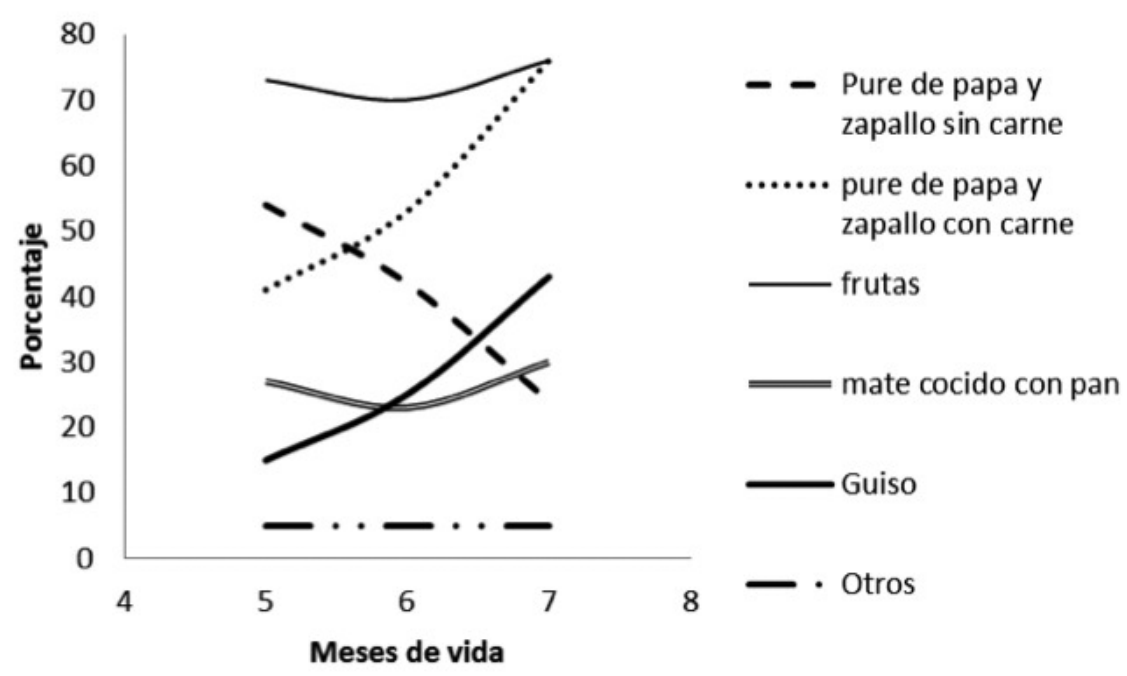


fue mayoritariamente puré de verduras, principalmente de zapallo o zanahoria; luego puré de frutas, donde predominó la manzana. La edad promedio de incorporación de la primera comida fue de 5 meses (20). Según la Encuesta Nacional de Nutrición y Salud (6) los alimentos incorporados tempranamente por mayor cantidad de niños fueron el yogur, los vegetales cocidos y los purés de frutas, seguidos por los jugos de frutas.

Un estudio realizado en Chile (25) mostró que 42,2\% de los lactantes iniciaban la alimentación complementaria con la introducción simultánea de alimentos de origen vegetal (cereales y/o fruta y/o verdura) y de origen animal (pollo y/o ternera), seguidos de 19,4\%, que la iniciaban con frutas. Estudios en la región amazónica de Brasil (13) mostraron una introducción precoz de alimentos ricos en carbohidratos junto con leche de vaca e ingestas irregulares de frutas, vegetales y carne. En los Andes de Bolivia (16) se observó que 39\% había iniciado la alimentación complementaria en los primeros tres meses, principalmente con papa y chuño (papa deshidratada); recibían cantidades limitadas de maíz, trigo, tarwi (leguminosa de alto valor proteico), arveja, haba y cebada, y una o dos cucharadas de caldo dos a tres veces al día.

Estos patrones de alimentación son habituales no sólo en muchos de los países de la región (26) sino que también se observan en muchos otros países en vías de desarrollo (27). Esto significa que un elevado porcentaje de niños consume precozmente papillas ricas en almidones caracterizadas por una baja densidad energética, escaso aporte de proteínas de alta calidad así como de minerales esenciales, como hierro, zinc y calcio, que no es lo indicado como primera alimentación complementaria.

Según las recomendaciones $(2,5,28)$ los alimentos complementarios deben introducirse a partir de los 6 meses, comenzando con cantidades pequeñas de alimentos, cuya frecuencia dependerá de su densidad energética. Se aconseja dar una variedad de alimentos para asegurarse de cubrir las necesidades tanto de macro como de micronutrientes e incluir, en lo posible, alimentos de origen animal así como frutas y verduras ricas en vitamina A diariamente. Es importante proveer dietas con un contenido adecuado de grasa.

Durante este período de transición, los lactantes deberían adaptarse gradualmente a la alimentación familiar hasta reemplazar completamente la leche materna ya que los niños serían fisiológicamente capaces de consumir los alimentos familiares recién al año de vida. Es de destacar que muchos niños se incorporaron a la mesa familiar desde los 5 meses de edad, siendo el guiso uno de los alimentos más frecuentemente consumidos. En este estudio se observó una introducción precoz y simultánea de alimentos nutricionalmente inadecuados, acompañada de una ingesta elevada de sodio, con el riesgo de una excesiva carga renal de solutos así como de hipertensión arterial a largo plazo. Por otra parte, también fue precoz la incorporación de alimentos con gluten (sémola, fideos), con los riesgos inherentes a la posibilidad de enfermedad celíaca.

En muchos casos se reemplazaba la leche con infusiones tales como el mate cocido, de escaso valor nutricional, además de contener polifenoles que interfieren con la utilización de minerales.

Las prácticas inadecuadas de alimentación en este período crítico pueden aumentar el riesgo de retraso del crecimiento y deficiencias nutricionales especialmente de hierro, y pueden tener efectos a largo plazo sobre la salud y el desarrollo mental (29).

Por otra parte, la incorporación precoz de alimentos complementarios puede desplazar a la leche materna, Ile- vando a una menor producción láctea, y por consiguiente, a una ingesta insuficiente de energía y nutrientes. Los lactantes están expuestos a microorganismos patógenos presentes en alimentos y fluidos, potencialmente contaminados, y por lo tanto, con mayor riesgo de diarreas y malnutrición; los riesgos de diarrea y alergias alimentarias se incrementan debido a la inmadurez intestinal.

Las papillas más frecuentemente consumidas son energéticamente inadecuadas, por lo que debería incorporarse una cantidad superior a la capacidad gástrica para cubrir las necesidades calóricas. Esto se debe a que en la elaboración de los purés, para lograr la textura deseada se agrega el caldo de la sopa de cocción de las verduras, en lugar de aceite.

En nuestro país, es habitual por razones culturales, que la primera opción entre los ingredientes utilizados para introducir alimentos sólidos sean la papa, el zapallo y la zanahoria, aportadores de hidratos de carbono y fibra (29). Si bien el hospital cuenta con un pediatra, éste no reside en el lugar, y solo atiende 2 veces por semana, por lo que se puede presumir que en las consultas se prioriza la atención de patologías de salud y que las indicaciones sobre la alimentación del niño son escasas.

\section{CONCLUSIONES}

Las propiedades nutricionales e inmunológicas de la leche materna son muy superiores a las de cualquiera de sus sucedáneos comerciales. En la población estudiada se observó un elevado porcentaje de niños alimentados desde su nacimiento con leche de vaca paralelamente a la lactancia materna, lo que originaría una disminución de la producción láctea y el consecuente desplazamiento de la lactancia natural. Por otra parte, la introducción temprana de alimentos complementarios de baja densidad energética y gran volumen (puré amarillo o de fruta) produciría saciedad, desplazamiento de la leche materna y por lo tanto una disminución de la ingesta total de energía. Fue escaso el aporte de alimentos proteicos de alta calidad y de minerales esenciales. La incorporación del niño a la mesa familiar fue temprana, con introducción precoz y simultánea de alimentos nutricionalmente inadecuados.

Esta situación podría tener efectos deletéreos tanto a corto como a largo plazo sobre la salud, el crecimiento físico y el desarrollo cognitivo del niño.

Estos resultados refuerzan los argumentos a favor de una sólida política nacional a favor de la lactancia materna así como de la introducción de alimentos complementarios adecuados en el momento oportuno. Sin embargo, esta es una tarea de gran complejidad que requiere enfoques múltiples a fin de ser efectivos (30). Dichas intervenciones deberían complementarse con las ya desarrolladas por diversos organismos públicos que actúan a nivel local en el área de la alimentación y nutrición.

\section{RESUMEN}

En Argentina existe escasa información sobre las características de la alimentación complementaria. El objetivo del presente trabajo fue determinar la edad de inicio y tipo de alimentos complementarios incorporados en la dieta de lactantes, en una población de escasos recursos socio-económicos. El estudio se realizó en el Hospital Distrital de Forres, Santiago del Estero, utilizando una encuesta estructurada. Las madres en etapa de lactancia fueron interrogadas durante las visitas periódicas de control de sus hijos. Se realizaron 240 encuestas, a los 5, 6 y 7 meses post-parto. La edad promedio de incorporación de alimentos fue 4,4 meses. Los primeros 
alimentos incorporados fueron: puré (papa y zapallo, con caldo o sopa), sopa (con sémola) y manzana. El $51 \%$ de los niños consumía algún tipo de papilla a los 5 meses. A los 7 meses, el $94 \%$ consumían papillas y el $62,5 \%$ tomaban mamadera. El $40 \%$ comía el guiso familiar (verduras, fideos y carne) y el $30 \%$, mate cocido con pan. Se observó incorporación temprana de alimentos con densidad energética y de nutrientes inadecuadas, ingesta precoz de gluten y elevada proporción de consumo de leche de vaca. Esta situación podría afectar el normal desarrollo de los niños de la población estudiada.

Palabras clave: lactancia materna; alimentos complementarios; nutrientes; densidad energética; leche de vaca.

Agradecimientos: El presente trabajo se realizó dentro de la Programación UBACyT, Proyectos B063 y 20020100100166.

\section{BIBLIOGRAFÍA}

1. Guía de alimentación de niños sanos de 0 a 2 años. Comité de Nutrición. Sociedad Argentina de Pediatría. Buenos Aires. Argentina. 2001. Disponible en: http://www.sap. org. ar/index.php?option=content\&task=view\&id=245\&/t ermid=26 Consulta: Febrero 2010.

2. Guías Alimentarias para la Población Infantil. Ministerio de Salud y Ambiente de la Nación. R. Argentina, 2006.

3. Macías SM, Rodríguez S, Ronayne de Ferrer PA. Leche materna: composición y factores condicionantes de la lactancia. Actualización. Arch Argent Pediatr 2006; 104: 423-30.

4. Caulfield LE, Esteban RA, Rivera JA, Musgrove P, Negro RE. Stunting, Wasting, and Micronutrient Deficiency Disorders. Chapter 28 Washington (DC): World Bank; 2006. Disponible en: http://files.dcp2.org/pdf/DCP/DCP28.pdf Consulta: Julio 2009.

5. Complementary feeding of young children in developing countries: a review of current scientific knowledge. World Health Organization, Geneva, 1998. Disponible en: http:// whqlibdoc.who.int/hq/1998/WHO_NUT_98.1.pdf Consulta: Octubre 2009.

6. La alimentación de los niños menores de dos años. Resultados de la Encuesta Nacional de Nutrición y Salud -ENNyS 2010. Buenos Aires: Ministerio de Salud, 2010. Disponible en: http://www.msal.gov.ar/promin/publicaciones/pdf/ la_alimentacion_de_los_ninos_menores_de_2_anos.pdf Consulta: Marzo 2011.

7. Albarenque S, Mas L, Ferreyra M, Marchisio M I, Gomila A, Armelini P. Lactancia materna y alimentación complementaria Arch Argent Pediatr 2005; 103: 257-58.

8. Gatica Cl, Méndez de Feu MC, e investigadores principales. Prácticas de alimentación en niños menores de 2 años. Arch Argent Pediatr 2009; 107: 496-503.

9. Jiménez $R$, Curbelo JL, Peñalver $R$. Relación del tipo de alimentación con algunas variables del crecimiento, estado nutricional y morbilidad del lactante. Colombia Médica 2005; 36 (Sup/ 3): 19-25.

10. González-Cossío T, Rivera-Dommarco J, Moreno-Macías $H$, Monterrubio E, Sepúlveda J. Poor Compliance with Appropriate Feeding Practices in Children under $2 y$ in Mexico. J Nutr 2006; 136: 2928-2933.

11. Garcia de Lima Parada CM, de Barros Leite Carvalhaes MA, Temer Jamas M. Prácticas de alimentación complementaria en niños dentro del primer año de vida. Rev Latino-am Enfermagem 2007; 15: 282-89.

12. Sánchez-Jaeger A, Barón MA, Solano Rodríguez L, Guerrero $A$, Díaz N. Prácticas inadecuadas de alimentación entre el $4^{\circ}$ y $6^{\circ}$ mes de vida y su asociación con el déficit nutricional en un grupo de lactantes venezolanos. Arch Latinoamer Nutr 2008; 58: 228-33.

13. Castro TG, Baraldi LG, Muniza PT, Cardoso MA. Dietary practices and nutritional status of 0-24-month-old children from Brazilian Amazonia. Public Health Nutr. 2009; 12: 2335-42.

14. Olaya G, Borrero ML. Propuesta para la formulación de pautas para la alimentación complementaria del niño lactante de 6 a 12 meses. Perspect Nutr Humana. 2009; 11:139-51.

15. Martínez Vázquez RI, Alvarado Ruiz GA, Sánchez Pérez MC, Blanco Loyola LA, Sánchez Jesús MM, Blázquez Martínez JU, Méndez Celayo S. Estudio de las pautas alimentarias para la introducción de alimentos complementarios y su diversidad a través de la incorporación a la dieta familiar. Rev Especialidades Médico-Quirúrgicas 2010; 15:114-24.

16. Cruz Agudo Y, Jones AD, Berti PR, Larrea Macías S. Lactancia materna, alimentación complementaria y malnutrición infantil en los Andes de Bolivia. Arch Latinoam Nutr 2010; 60: 7-14

17. UNICEF. Informe Anual de Actividades. Argentina 2010. Salud y Nutrición. Disponible en: http://www.unicef.org/ argentina/spanish/Informe_Anual_2010.pdf Consulta: Marzo 2011.

18. Centro Interamericano para el Desarrollo del Conocimiento en la Formación Profesional ANEXO I - INDICE DE NIVEL SOCIOECONÓMICO. Disponible en: http://wWw.cinterfor.org.uy/public/spanish/region/ampro/cinterfor/temas/ youth/doc/jov_mer/par/iii/index.htm Consulta: Junio 2010.

19. WHO Collaborative Team on the Role of Breastfeeding in the Prevention of Infant Mortality. Lancet 2000; 55: 451-5.

20. Bove MI, Cerruti F. Encuesta de lactancia, estado nutricional y alimentación complementaria. UNICEF, Uruguay, 2007. Disponible en: http://www.unicef.org/uruguay/ spanish/uy_media_Encuesta_de_lactancia.pdf Consulta: Marzo 2009.

21. Calvo EB, Gnazzo N. Prevalence of iron deficiency in children aged 9-24 months from a large urban area of Argentina. Am J Clin Nutr. 1990; 52: 534-40.

22. Morasso M, Molero J, Vinocur P, Acosta L, Paccussi N, Raselli S, Falivene $G$, Viteri F. Deficiencia de hierro y vitamina A y prevalencia de anemia en niños y niñas de 6 a 24 meses de edad en Chaco, Argentina. Arch Latinoam Nutr. 2003; 53:21-7.

23. Escribano J, Luque $V$, Ferre $N$, Zaragoza-Jordana $M$, Grote $V$, Koletzko B, Gruszfeld D, Socha P, Dain E, Van Hees JN, Verduci $E$, Closa-Monasterolo R. Increased protein intake augments kidney volume and function in healthy infants Kidney Internacional 2011; 79: 783-790.

24. Reyes C, Atalah E, Castillo C. Efectividad del Programa de Lactancia. Comisión Nacional de Lactancia Materna. Ministerio de Salud. Chile. 2005. Disponible en: http://www. redsalud.gov.cl/archivos/informe_lactancia_oct_2005.pdf Consulta: Septiembre 2012

25. Santamaría Orleans A. Hábitos alimentarios de los lactantes españoles y chilenos. Tesis Doctoral. Universidad de Barcelona. 2005.

26. Lutter CK. Growth and complementary feeding in the Americas. Nutr Metab Cardiovas Dis. 2012; 22: 806-12.

27. Ronayne de Ferrer PA, Greco CB, Binaghi JM. Complementary feeding in developing countries. Editor: Rosana 
Filip. Multidisciplinary Approaches on Food Science and Nutrition for the XXI Century. Kerala, India, Transworld Research Network, 2011, p. 245-273.

28. PAHO and WHO (Pan American Health Organization and World Health Organization). Guiding Principles for Complementary Feeding of the Breastfed Child. Washington, DC: PAHO and WHO. 2003. Disponible en: http://WWW. who. int/nutrition/publications/guiding_principles_compfeed- ing_breastfed.pdf Consulta: Marzo 2010.

29. Macias SM, Rodriguez S, Ronayne de Ferrer PA. Características de una alimentación complementaria adecuada. Actualización. Rev Soc Argentina Nutr. 2007; 8: 113-9.

30. Dewey KG, Brown KH. Update on Technical Issues Concerning Complementary Feeding of Young Children in Developing Countries and Implications for Intervention Programs. Food Nutr. Bull. 2003; 24:5-28. 